\title{
Application of Acoustic Emission Technique to Monitor the Viscosity of Single Grade Diesel Engine Lubricant Oil.
}

\begin{abstract}
This paper presents an investigation of the capability of the acoustic emission (AE) technology in the processes of monitoring and detecting the variation of diesel engine lubricant viscosity via in-situ operation. The investigations were conducted on an 8.5-hp single cylinder of four strokes with a water cooling diesel engine. One single grade engine lubricant oil condition has been put to the test; namely, SAE 40. The lubrication condition in the diesel engine was successfully monitored based on the AE signatures generated by the interaction between the piston surface and the layer of the lubricant during the time that the engine was in operation. The generated AE signatures were captured using an AE wide band transducer that was bonded outside the engine block and located at the lower part of the bottom dead centre (BDC). The captured waveform signatures were then analysed using the MATLAB software. The results of the experimental works show that the statistical analysis parameters including the root-mean-square (rms), maximum amplitude and AE energy values are capable of distinguishing the variant of the viscosity. The AE technique can assist the owner of the diesel engine in determining the diesel engine lubricant oil condition before replacing it.
\end{abstract}

Keyword: Diesel Engine Lubricant, Viscosity, Acoustic Emission, Maximum Amplitude, Root-Mean-Square, AE Energy 